\title{
Pricing Arithmetic Asian Options under the CEV Process
}

\section{Valorización de la Opción Asiática Aritmética bajo el Proceso de Variación Elástica Constante (CEV Process)}

\author{
Bin Peng ${ }^{1}$ \\ Fei Peng ${ }^{2}$
}

Received date: June 30, 2010

Accepted date: August 15, 2010

\begin{abstract}
This paper discusses the pricing of arithmetic Asian options when the underlying stock follows the constant elasticity of variance $(\mathrm{CEV})$ process. We build a binomial tree method to estimate the CEV process and use it to price arithmetic Asian options. We find that the binomial tree method for the lognormal case can effectively solve the computational problems arising from the inherent complexities of arithmetic Asian options when the stock price follows CEV process. We present numerical results to demonstrate the validity and the convergence of the approach for the different parameter values set in CEV process.
\end{abstract}

Keywords: Exotic options, arithmetic Asian options, binomial tree method, CEV process

\section{Resumen}

Este artículo discute la valoración de las opciones asiáticas aritméticas cuando las acciones subyacentes siguen el proceso de la variación elástica constante (modelo CEV, por sus siglas en inglés). Construimos un método de árbol binómico para estimar el proceso CEV y se usó para valorar las opciones asiáticas aritméticas. Hallamos que el método de árbol binómico puede resolver eficientemente los problemas de computación que se dan por las complejidades inherentes a las opciones asiáticas aritméticas cuando el precio del mercado sigue el modelo CEV. Aquí presentamos resultados numéricos que demuestran la validez y la convergencia del enfoque para los diversos parámetros de valoración establecidos en el proceso CEV.

Palabras claves: Opciones exóticas, opciones asiáticas aritméticas, método de árbol binómico, proceso CEV

* This paper is supported by the National Natural Foundation of China (NO: 71002098).

1. Ph. D. in Finance. School of Business, Renmin University, Beijing, P. R., China. <Pengbin01@hotmail.com>.

2. Ph. D. in Computer Engineering. Electrical \& Computer Engineering, University of British Columbia, Vancouver, Canada. $<$ feip@ece.ubc.ca>. 


\section{INTRODUCTION}

Asian options are the popular financial tool in the over-the-counter market, whose payoffs depend on some form of averaging of the underlying stock price during some specific period. There are two kinds of Asian options: arithmetic Asian options and geometric Asian options. At present, arithmetic Asian options have become increasingly prevalent in the over-the-counter market. Kernna \& Vorst (1990) provided the accurate solution for European arithmetic Asian options. Since then closed-form solutions and numerical methods have been proposed to handle American or other more complex Asian options. Most of the published investigations on arithmetic Asian options assume that the underlying stock follows lognormal distribution process. As the underlying assumption of the Black-Scholes model (1973), it has some drawbacks. Empirical studies have shown that stock prices are generally unlikely to be lognormally distributed. When the Black-Scholes model is used to price stock options, certain biases, such as the strike price bias (volatility smile), persist because there lies in the negative correlation between stock price changes and volatility changes (Macbeth \& Merville, 1980). The CEV option-pricing model, originally developed by Cox (1975), incorporates this negative correlation. It is instructive to apply the CEV process to arithmetic Asian options.

The binomial tree method (BTM), first proposed by Cox, Ross \& Rubinstein (1979), is the most simple and powerful technique to pricing many complex exotic options in contrast to the Partial Differential Equation (PDE) method and other numerical methods such as Monte-Carlo simulated method (Duan, 1995) and lattice method (Boyle, 1986, Ritchken \& Trevor, 1999). Cox (1976) and Phelimp \& Tian (1999) published the pricing of the standard European option, barrier and lookback options when the underlying stock price follows the CEV process. There is, however, little work on Asian option.

The objective of this paper is to study the application of the binomial tree approach to arithmetic Asian options when the stock price evolves as a CEV process.
The outline of the rest of the paper is as follows: the first section describes the constant elasticity of variance (CEV) process The following section develops the binomial tree method to the valuation of arithmetic Asian option under the CEV process, and the convergence of the binomial tree method is indicated using numerical example. Conclusions are presented in the final section.

\section{THE CONSTANT ELASTICITY OF VARIANCE (CEV) PROCESS}

The Black \& Scholes (1973) option pricing model assumes that the underlying stock pays no dividends during the option period, and the underlying stock price follows the lognormal diffusion process:

$$
d S=\mu S d t+\sigma S d z
$$

where $d z$ is wiener process. The assumption means that the percentage change in the stock price $d S / S$ over the small interval $d t$ is normally distributed with mean $\mu d t$ and instantaneous variance $\sigma^{2} d t . \mu$ ad $\sigma$ represent the expected yield rate and volatility, respectively.

It is known that the lognormality assumption does not hold empirically for stock prices or stock indices. Alternative stochastic processes have been studied and applied to option pricing .For example, Cox (1975) and Cox \& Ross (1976) studied a general class of stochastic processes known as the constant elasticity of variance (CEV) process.

Denote the stock price at an instant of time $t$ as $S$, and let the change in the stock price over the next small increment of time, $d t$, be denoted as $d S$. Then, for constants $\mu, a$, the family of constant elasticity of variance diffusion process can be described by the stochastic differential equation (Cox \& Ross, 1976):

$$
d S=\mu S d t+\sigma S^{a / 2} d z
$$

where $a$ is an elasticity factor, and $0 \leq a<2$. The process with $0 \leq a<2$ has some economic implications. We 
assume that all the companies lie in the fixed costs of no concern with the running trackrecord. When stock price decreases, the company trackrecord decreases, and fixed costs have the effect on increasing volatility; when stock price increases, fixed costs have the effect on decreasing volatility.

In the limiting case when $a=2$, the instantaneous variance of return is a constant, $\sigma^{2}$, and the stochastic process generating returns is a lognormal diffusion process, the process assumed in the equation (1), implying that lognormal diffusion process is a special case of CEV process. Following Cox (1975), we restrict the range of $a$ to the interval $[0,2]$. The process with $a<0$ does not have reasonable economic properties since the volatility explodes as we approach the origin and the stock price can become negative.

Equation (2) is based on nature's probability measure, the P-measure. In order to price securities under the CEV process, we first transform the stochastic process in (1) to the Q-measure under which the deflated price process of all securities is martingales. In this case, the accumulated money market account is used as the numéraire. Using the martingale measure theory, under the Q-measure, the revised process resembles (1) except that the risk-free rate $r$ (here assumed constant) replaces $\mu$.

$$
d S=r S d t+\sigma S^{a / 2} d \tilde{z}
$$

where

$$
d \tilde{z}=d z+\frac{\mu-r}{\sigma} S^{1-a / 2} d t
$$

\section{BINOMIAL TREE METHOD FOR THE ARITHMETIC ASIAN OPTION UNDER THE CEV PROCESS}

In this section, we extend the vanilla arithmetic Asian option when the underlying stock price follows arithmetic Brownian motion and further examine the arithmetic Asian option valuation with the CEV process, in which volatility is not constant, changing with the stock price. It is difficult to derive the analytical solution of the arithmetic Asian option valuation with the CEV process; we develop a discrete approximation for the CEV process using the binomial tree method to evaluate the arithmetic Asian option valuation with the CEV process.

We assume the stock pays no dividends. The increasing rate of the stock price is $u$, the increasing probability of the stock price is $p$, and the decreasing rate of the stock price is $d$. And we have $u>(1+r) \Delta \mathrm{t}>1$, $d<1<(1+r) \Delta t . r$ is risk-free rate and it is constant and positive. If the current stock price is $S_{0}$, the stock price at the end of one period $\Delta t$ will thus be either $u \times S_{0}$ or $d \times S_{0}$. Following the binomial process, the stock can take three possible values after two periods $2 \Delta t: u^{2} S_{0}$, $u d S_{0}, d^{2} S_{0}$; at the end of three periods $3 \Delta t$, the stock has four possible values: $u^{3} S_{0}, u^{2} d S_{0}, u d^{2} S_{0}, d^{3} S_{0}$. At the end of $i$ periods $i \Delta t$, the stock has $i+1$ possible values (Cox, Ross \& Rubinstein, 1979):

$$
S(i+1, j)=S_{0} u^{j} d^{i-j} \quad j=0,1,2 \ldots N
$$

At the expiration date $T=N \Delta t$, the stock has $N+1$ possible value

Such the stock price movement process is illustrated in Figure. 1 for the case when $i=3$.

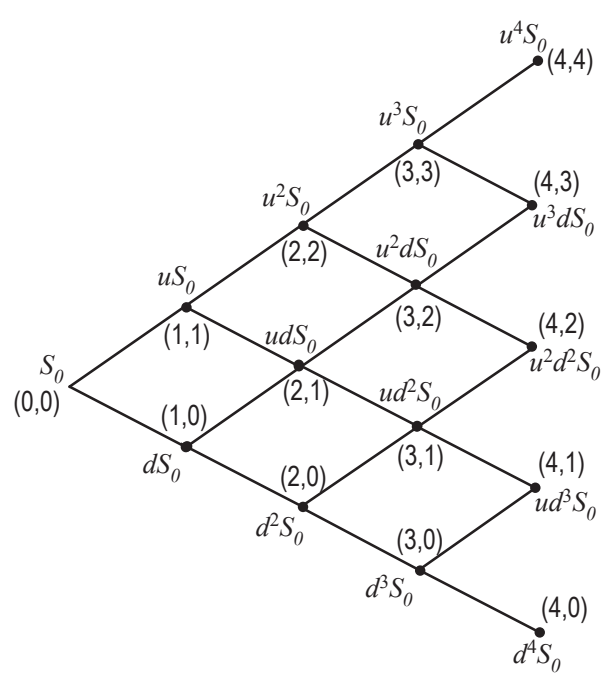

Figure 1. Binomial Tree Structure 
In the following section, we develop a discrete approximation for the $\mathrm{CEV}$ process using the binomial tree method. We assume that the stock price dynamics are expressed in terms of the Q-measure. We transform the variable $S$ so that the transformed process has constant volatility. We let

$$
X=X(t, S)
$$

Applying Ito's Lemma, the stochastic differential equation for $X$ is

$$
d X=h(t, S) d t+\frac{\partial X}{\partial S} \sigma S^{a / 2} d z
$$

where $h(t, S)=\frac{\partial X}{\partial t}+r S \frac{\partial X}{\partial S}+\frac{1}{2} \sigma^{2} S^{a} \frac{\partial^{2} X}{\partial S^{2}}$

To ensure that process (5) has constant volatility, we must find a transformation such as this:

$$
\beta=\frac{\partial X}{\partial S} \sigma S^{a / 2}
$$

For some positive constant $\beta$, this is equivalent to

$$
\frac{\partial X}{\partial S}=\frac{\beta}{\sigma} S^{-a / 2}
$$

For $a \neq 2$, the transformation is given by

$$
X=\frac{\beta S^{1-a / 2}}{\sigma(1-\mathrm{a} / 2)}
$$

For $a=2$, the appropriate transformation is given by

$$
X=\frac{\beta}{\sigma} \log S
$$

For the case with $a \neq 2$, applying the equation (8), the transformed equation (5) becomes

$$
d X=\left\{r\left(1-\frac{a}{2}\right) X-\frac{a \beta^{2}}{4\left(1-{ }_{2}^{a}\right) X}\right\} d t+\beta d \tilde{z}
$$

The transformed process (10) has constant volatility, which lays the foundation on the application of the Binomial Tree method to evaluating the arithmetic Asian option. However, we find that the yield term in the transformed process tends to the infinity when $\mathrm{X} \rightarrow 0$ (for the only exception when $a=0$ ).

$$
\lim _{X \rightarrow 0}\left[r\left(1-\frac{a}{2}\right) X-\frac{a \beta^{2}}{4(1-(a / 2) X}\right]=\infty(a \neq 0)
$$

It is difficult for us to value the arithmetic Asian option using the binomial tree method, so we first need to construct the following discrete approximation

$$
\begin{aligned}
& t_{0}, t_{0}+\Delta t, \ldots \ldots, t_{0}+N \Delta t \\
& X_{0}, u X_{0}, d X_{0}, u^{2} X_{0}, u d X_{0}, d^{2} X_{0}, \ldots \ldots
\end{aligned}
$$

Where $\left(t_{0}, X_{0}\right)$ is the starting point of the binomial process, $u>0, d=1 / u . \Delta t$ is the increment in time, and $X_{0}$ is related to the initial stock price $S_{0}$ through the transformation by

$$
X_{0}=\frac{\beta S_{0}^{1-a / 2}}{\sigma(1-a / 2)}
$$

However, the transformed discrete process cannot solve the boundary problem at zero. A solution is to use inverse transformation of (7) defined by

$$
S=\left\{\left[\begin{array}{cl}
{\left[\frac{\sigma\left(1-\frac{a}{2}\right) X}{\beta}\right]^{\frac{1}{1-a / 2}}} & \text {, if } X_{j}>0 \\
0, & \text { otherwise }
\end{array}\right.\right.
$$

This new discrete approximation is represented as

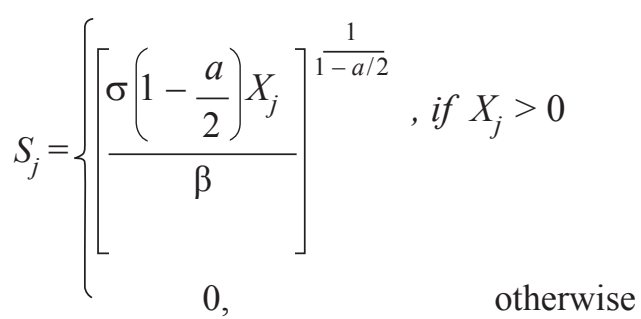


The formulae for $p, u$ and $d$ are as follow (see Hull, 2002)

$$
u=b+\sqrt{b^{2}-1} d=1 / u p=\frac{e^{r \Delta t}-d}{u-d}
$$

where $b=\left(e^{r \Delta t}+e^{(r+\sigma 2) \Delta t}\right) / 2^{1}$

We further write $(i, j)$ for the node of the binomial tree where the underlying stock has a price $S(i, j)(j=0,1, \ldots . ., i$; $i=0,1, \ldots \ldots, N)$. Note that $i-j$ is the down steps and $j$ is the up steps taken by the underlying stock price. Then, we use the index $k(k=0,1, \ldots . . i-j)$ and $h(h=0,1, . j-1)$ respectively to specify the lowest and the highest layer of horizontal nodes reached by the underlying stock price after $i$ time steps. In other words, $k=1$ means that the stock with current price $S(i, j)$ has registered minimum price equal to $S(1,0) h=1$; this means that the stock with current price $S$ has registered maximum price equal to $S(1,1)$. Clearly, the underlying stock price at node $(i, j), S_{i}^{j}$ is greater than $S_{1,0}$; at node $(k, 0)$ and less than $S(h, h)$ at node $(h, h)$. In addition, $S(i, j)$ reach $S(k, 0)$ by the total of $j$ down steps. Analogously, $S(i, j)$ touches $S(h, h)$ by the total of $i-j$ up steps. Hence, the maximum or minimum state sum of nature $(i, j)$ can be represented as follows (see Figure 1):

$$
\begin{gathered}
\max (i, j)=\sum_{p=j}^{i}(p, j)+\sum_{h=0}^{j-l}(h, h) \\
\min (i, j)=\sum_{q=i-[j / 2]}^{i}[q, q-(i-j)]+\sum_{k=0}^{j-l}(k, 0)
\end{gathered}
$$

where $L_{0}$ returns the lower integer is closest to a real number.

We then define arithmetic mean as follow:

$$
A\left(t_{0}, t_{\mathrm{k}}\right)=\left[S\left(t_{1}\right)+\left(t_{2}\right)+S\left(t_{3}\right)+\ldots .+S\left(t_{\mathrm{k}}\right)\right] / k
$$

1 To ensure that arithmetic Asian option prices based on different value of a are broadly comparable, the value of $\sigma$ in each model is readjusted so that the initial instantaneous volatility is the same across different models. Let $\sigma_{0}$ be the instantaneous volatility for the lognormal model $(\mathrm{a}=2)$. Following MacBeth and Merville (1980), the value of $\sigma$ to be used for models with other $a$ values is adjusted to be $\sigma=\sigma_{0} S^{1-a / 2}$ such that $\sigma_{0}$ is the instantaneous volatility for the lognormal model $(a=2)$ where

$$
\begin{aligned}
& 0=t_{0}<t_{1}<t_{2}<\ldots .<t_{k}<t_{k+1}<\ldots . . \mathrm{t}_{\mathrm{N}}=T \\
& A\left(t_{0}, t_{\mathrm{N}}\right)=\left(S_{1}+S_{2}+\ldots . .+S_{N}\right) / N
\end{aligned}
$$

Hereby we can easily get the maximum arithmetic average reaching node $(N, j)$

$A_{N, j}^{\max }\left(t_{0}, t_{\mathrm{N}}\right)=S_{0}\left(\frac{\mu\left(1-u^{j}\right)}{1-u}+\frac{u^{j} d\left(1-d^{N-j}\right)}{1-d}\right) / N$

The minimum arithmetic average reaching node $(i, j)$ is

$A_{N, j}^{\min }\left(t_{0}, t_{\mathrm{N}}\right)=S_{0}\left(\frac{d^{N-j} \mu\left(1-u^{j}\right)}{1-u}+\frac{d\left(1-d^{N-j}\right)}{1-d}\right) / N$

where

$$
j=0,1,2, \ldots \ldots, N \text {. }
$$

Based on the definitions of standard arithmetic Asian call option (Hull, 2002), the arithmetic Asian call option boundary condition is

$$
f(N, j)=\operatorname{Max}\left(0, A_{N, j}^{\operatorname{Max}}-K\right)
$$

Based on the definitions of standard arithmetic Asian put option (Hull, 2002), the arithmetic Asian put option boundary condition is

$$
f(N, j)=\operatorname{Max}\left(0, K-A_{N, j}^{\operatorname{Min}}\right)
$$

where $K$ is strike price.

The price of arithmetic Asian call and put options under $\mathrm{CEV}$ process is calculated using the binomial tree option pricing equation

$$
\begin{aligned}
& f(i, j)=e^{r \Delta t}[p f(i+1, j+1)+(1-p) f(\mathrm{i}+l, j)] \\
& 0 \leq \mathrm{i} \leq N-1,0 \leq j \leq i
\end{aligned}
$$


The numerical example on Table 1 reports prices of arithmetic Asian call and put options under CEV process using the binomial tree method. The focus of this table is the convergence properties of the binomial tree method. We assume that the above-mentioned arithmetic Asian option is European arithmetic Asian option on a non-dividend paying stock. The current stock prices are 110,115 , or 120 , strike price is 115 , and the term to maturity is six months. The risk-free rate $r$ is 0.1 per annum, the number of time steps $(N)$ is 300 or 350 , and the instantaneous volatility (of the percentage change in stock price) is 0.8 per annum. We employ three $a$ values to show its effect on arithmetic Asian option prices. These represent the absolute model $(a=0)$, the square root model $(a=1)$, and the lognormal model $(a=2)$. In the case of lognormal model, the standard binomial method is used (Cox, 1976).

From the result shown on Table 1, it is clear that prices from the binomial tree method converge rapidly with all $a$ values. The convergence of arithmetic Asian option prices for the absolute model and the square root model are comparable to that of arithmetic Asian option prices for the lognormal model. The results using the binomial tree approach developed here are consistent with the results given by the pricing formulae under the continuous situation (Kernna \&Vorst, 1990).

\section{CONCLUSION}

This paper researches on the pricing of arithmetic Asian option when the underlying stock follows the constant elasticity of variance (CEV) process. A binomial tree method was developed to approximate the $\mathrm{CEV}$ process and we used it to value arithmetic Asian call and put options. The method is simple to use and has satisfactory convergence for the pricing of exotic arithmetic Asian option. We demonstrate that the prices of the exotic option for the CEV process can deviate significantly from the standard option for the lognormal process, even after proper adjustment for volatility difference. In addition, for arithmetic Asian option that depends on the extreme of the process, the prices are quite sensitive to the specification of the process.

Table 1.

Convergence of arithmetic Asian options under CEV process

\begin{tabular}{|c|c|c|c|c|c|c|c|}
\hline \multirow{2}{*}{ Current Stock Price } & \multirow{2}{*}{ Strike Price } & \multicolumn{2}{|c|}{ Time Steps $N=300$} & \multicolumn{4}{|c|}{ Time Steps $N=350$} \\
\hline & & $a=0$ & $a=1$ & $a=2$ & $a=0$ & $a=1$ & $a=2$ \\
\hline \multicolumn{8}{|c|}{ Part A: arithmetic Asian call option } \\
\hline 110 & 115 & 7.1225 & 7.1235 & 7.1237 & 7.0680 & 7.0690 & 7.0692 \\
\hline 115 & 115 & 12.4186 & 12.4196 & 12.4198 & 12.3616 & 12.3627 & 12.3629 \\
\hline 120 & 115 & 17.7146 & 17.7157 & 17.7160 & 17.6552 & 17.6563 & 17.6566 \\
\hline \multicolumn{8}{|c|}{ Part B: arithmetic Asian put option } \\
\hline 110 & 115 & 9.7225 & 9.7233 & 9.7235 & 9.7678 & 9.7686 & 9.7688 \\
\hline 115 & 115 & 5.1921 & 5.1929 & 5.1931 & 5.2394 & 5.2403 & 5.2405 \\
\hline 120 & 115 & 0.8720 & 0.8727 & 0.8729 & 0.8795 & 0.8801 & 0.8803 \\
\hline
\end{tabular}




\section{References}

Black, F., \& Scholes, M.(1973). The Pricing of Option and Corporate Liabilities. Journal of Political Economy, 81, 637-659.

Boyle, P. P. (1986). Option Valuation Using a Three-jump Process. International Option Journal, 3, 7-12.

Cox, J. C. (1975). Notes on option pricing I: Constant elasticity of variance diffusions. Unpublished note. Stanford University, Graduate School of Business.

Cox, J. C. (1996). The Constant Elasticity of Variance Option Pricing Model. Journal of Portfolio Management, 22, 15-17.

Cox, J. C., \& Ross, S. A. (1976). The Valuation of Options for Alternative Stochastic Processes. Journal of Financial Economics, 3, 145-166.

Cox, J. C., Ross, S. A., \& Rubinstein, M. (1979). Option Pricing: A Simplified Approach. Journal of Financial Economics, 3, 229-264.

Duan, J. C. (1995). The GARCH Option Pricing Model. Mathematical Finance Journal, 5, 13-32.
Duffie, D. (1996). Dynamic Asset Pricing Theory. New Jersey: Princeton University Press.

Hull, J. C. (2002). Options, Futures and other Derivatives. (5th ed.). New York: Prentice Hall.

Kernna, A. G. Z., \& Vorst, A. C. F. (1990). A Pricing Method for Options Based on Average Asset Values. Journal of Banking and Finance, 14, 113-129.

MacBeth, J. D., \& Merville, L. J. (1980). Tests of the BlackScholes and Cox Call Option Valuation Models. Journal of Finance, 35, 285-301.

Phelimp, B., \& Yisong Tian (1999). Pricing Lookback and Barrier Options under the CEV Process. Journal of Financial and Quantitative Analysis, 34, 242-264.

Ritchen, P., \& Trevor, R. (1999). Pricing Options under Generalized GARCH and Stochastic Volatility Process. Journal of Finance, 54, 377-402. 